\title{
NEUROLEPTIC MALIGNANT SYNDROME IN PATIENTS WITH SCHIZOPHRENIA
}

\author{
Herlinda Luhulima ${ }^{a}$, Ferdy Royland Marpaung ${ }^{b^{*}}$

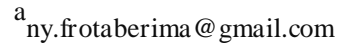

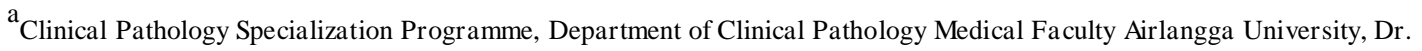 \\ Soetomo Hospital, Mayjen. Prof.Dr.Moestopo st no.6-8 Surabaya, 60286, Indonesia. \\ ${ }^{b}$ Department of Clinical Pathology, Medical Faculty Airlangga University, Dr. Soetomo Hospital, Mayjen. Prof.Dr.Moestopo st \\ no.6-8 Surabaya, 60286, Indonesia.
}

\begin{abstract}
Introduction: Neuroleptic Malignant Syndrome (NMS) is a rare and life treathening idiosyncratic reaction that can be caused by neuroleptic drugs with manifestation of fever, muscular rigidity, changes in mental status and autonomical dysfunction. NMS can happen soon after neuroleptic drugs initiation or after dosage increase. Ninety percent of cases can happen within 10 days and usually progress over 24-72 hours. Nevertheless, NMS can happen after years of therapy, therefore, antipsychotic administration requires a creatinine phosphokinase (CPK) examination. Elevated CPK level is a sign of rhabdomyolisis, secondary to muscular rigidity, caused by dopamine receptor blockage in the corpus striatum and nigrostriatal pathway. Establishing the diagnosis of NMS is based on The Diagnostic and Manual of Mental Disorder, fourth edition (DSM-IV) and Levenson's clinical criteria.

Case: A 27-year-old female presented with complaints of fever. History of schizophrenia for 12 years. Physical examination showed temperature $37.6^{\circ} \mathrm{C}$; catatonic (+) ; muscular rigidity (+) ; blood pressure $130 / 90 \mathrm{mmHg}$; pulse rate 118x/minute ; GCS E3 V1 M5. Hematological examination results: WBC 11x10³/uL; and Plts $131 \times 10^{3} / \mathrm{uL}$. Chemical chemistry examination result : AST $294 \mathrm{IU} / \mathrm{L}$; ALT $183 \mathrm{IU} / \mathrm{L}$; LDH 447 ; BUN $28 \mathrm{mg} / \mathrm{dL}$; serum creatinine $2.01 \mathrm{mg} / \mathrm{dL}$; hematuria ; proteinuria ; CPK 1,183.1 U/L.
\end{abstract}

Conclusion: A schizophrenia patient on initiation, when increasing the dosage needs to be monitored with CPK to prevent the risk of neuroleptic malignant syndrome.

Keywords: Neuroleptic Malignant Syndrome; creatinine phosphokinase; schizophrenia 


\section{Introduction}

The introduction of antipsychotic drugs in the mid-1950s revolutionized the treatment of schizophrenia and other psychotic disorders. The side effects of antipsychotic drugs are very important to know, given the long-term use of these drugs. Significant side effects of antipsychotic drugs to patients are often unpleasant and rarely life-threatening. An exception is Neuroleptic Malignant Syndrome (NMS) (1).

Neuroleptic Malignant Syndromesyndrome is athat occurs as a result of serious complications from the use of antipsychotic drugs. The characteristics of NMS are hyperthermia, rigidity, autonomic dysregulation and altered consciousness.

Neuroleptic Malignant Syndrome is a life-threatening complication that can occur at any time during antipsychotic treatment, but is rare. Motor and behavioral symptoms include muscle rigidity, dystonia, akinesia, muteness, obtundation and agitation. Autonomic symptoms include high fever, sweating, increased pulse and blood pressure. Laboratory findings include elevated white blood cell counts, elevated creatinine phosphokinase, liver enzymes, myoglobinuria, and renal failure may occur.

Diagnostic criteria for NMS based on the Diagnostic and Statistical Manual of Mental Disorders, fourth edition (DSM-IV) are severe muscle rigidity disorders, elevated temperature, and other associated symptoms (diaphoresis, elevated blood pressure, tachycardia, incontinence, dysphagia, mutism, tremor, changes in level of consciousness to coma, leukocytosis, increased creatinine phosphokinase as a result of muscle stiffness.

Based on Levenson's clinical criteria, diagnostics based on three major criteria or two major criteria and four minor criteria Major criteria: fever, rigidity, increased creatinine phopokinaseMinor criteria: tachycardia, increased blood pressure, changes in level of consciousness, diaphoresis, leukocytosis(2). 
2. Case

Female, 27 years old, with fever. Patient was referred from Menur Hospital. Patient complained of fever since 3 days of SMRS. Since 10 days patients treated at Menur Hospital, due to changes in patient behavior ( likes to laugh alone and get angry). During treatment at Menur Hospital, the patient received Risperidone 2x2mg and Clozapine 1x25mg. Fever goes up and down, the patient does not want to eat and drink. The patient did not vomit, did not have diarrhea, bowel and bladder were not disturbed. Patient had a schizophrenia since 12 years ago with Haloperidol, Trihexyphenidyl, and Depakote as her drugs for therapy. Patient had no hypertension and also diabetes.

\section{Examination history and patient hospitalization:}

- March 15, 2019: Clinical chemistry examination of the patient showed increased levels of SGOT and SGPT. Complete blood count showed an increase in leukocytes. The patient was then hospitalized, and was tested for IgM anti-HAV, anti-HCV, serial SGOT and SGPT tests, Total Billirubin and Direct Billirubin tests. It is planned to examine Creatinine Phospokinase (CPK) to establish the diagnosis of Neuroleptic Malignant Syndrome.

- March 16, 2019: Monitoring for signs of NMS, urine production. Meningeal signs and neck stiffness were examined, and the results were (-). The patient's neurological status was obtained: E4-M5-VX. The patient's antipsychotic therapy was postponed.

- March 20, 2019: CPK examination outside the Department of Clinical Pathology, RSUD Dr. Soetomo is 1183.1 U/L. Catatonic was still found in the patient.

- March 21, 2019: Complete blood count revealed an increase in WBC (10.64 $\mathrm{x} 10^{3} / \mathrm{uL}$ ). Clinical chemistry examination revealed an increase in ALT (612 IU/L); AST (181 IU/L); LDH (447 U/L). Urine examination found erythrocytes $2+$; leukocytes 2+; albumin 2+; ketones $2+$. 


\section{Physical examination:}

General condition : E3-V1-M5

$$
\text { Catatonic (+) }
$$

Vital signs : BP 130/90 mmHg; HR 118x/minute; RR 29x/minute; $t 37.6^{\circ} \mathrm{C}$

Head/neck : anemic conjunctiva -/-, jugular distension -/-

Thorax : cast and pulmo within normal limits

Abdomen : sociable, bowel sounds (+) normal, spleen and liver within normal limits.

Extremities : warm, CRT $<2$ seconds, edema $-/-$

\section{Supporting Examination:}

Table 1. Hematological Examination

\begin{tabular}{|c|c|c|c|c|c|c|}
\hline Parameter & $03 / 15 / 19$ & $03 / 21 / 19$ & $03 / 25 / 19$ & $03 / 28 / 19$ & $04 / 09 / 19$ & Referral \\
\hline \multirow[t]{2}{*}{ Hb (g/dL) } & 12.7 & 13.2 & 12.4 & 11.4 & 13.1 & L 13.3-16.6 \\
\hline & & & & & & P $11.0-14.7$ \\
\hline RBC (10\%/uL) & 4.34 & 4.60 & 4.50 & 3.93 & 4.37 & $3.69-5.46$ \\
\hline \multirow[t]{2}{*}{ HCT (\%) } & 38.3 & 40.3 & 40.6 & 34.8 & 40.8 & L $41.3-52.1$ \\
\hline & & & & & & P $35.2-46.7$ \\
\hline MCV (fL) & 88,2 & 87.6 & 90.3 & 88.5 & 93.4 & $86.7-102.3$ \\
\hline MCH (pg) & 29.3 & 29.8 & 27.6 & 29.0 & 30.0 & $27.1-32.4$ \\
\hline $\operatorname{MCHC}(\mathrm{g} / \mathrm{dL})$ & 33.2 & 34.0 & 30.6 & 32.8 & 32.1 & $29.7-33.1$ \\
\hline RDW (\%) & 12.2 & 12.1 & 14.2 & 39.6 & 14.7 & $12.2-14,8$ \\
\hline PIt $\left(10^{3} / u L\right)$ & 131 & 328 & 224 & 151 & 200 & $150-450$ \\
\hline $\mathrm{WBC}\left(10^{3} / \mathrm{uL}\right)$ & 11.0 & 10.64 & 10.57 & 6.71 & 6.49 & $3.37-10$ \\
\hline Count leukocyte & & & & & & Eo 0.6-5.4 \\
\hline type $(\%)$ & & & & & & Baso 0.3-1.4 \\
\hline Eo /Baso/Neut/ & 4.0/0.4/71.4/2 & $1.1 / 0.5 / 71$ & $1.0 / 0.1 /$ & $4.2 / 0.4 /$ & $1.7 / 0.6 /$ & Neut 39.8-70.5 \\
\hline \multirow[t]{3}{*}{ Lymph/Mono } & $0.3 / 5.5$ & $2 / 20.1 / 7.6$ & $77.9 / 12.5 /$ & $71.4 /$ & $67.8 /$ & Lymph \\
\hline & & & 7.6 & $19.5 / 4.5$ & $22.3 / 7.6$ & 49.9 \\
\hline & & & & & & Mono 4.3-10.0 \\
\hline
\end{tabular}


Table 2. Clinical Chemistry Examination

\begin{tabular}{|c|c|c|c|c|c|c|c|}
\hline Parameter & $03 / 15 / 19$ & 03/19/19 & 03/21/19 & $03 / 25 / 19$ & $04 / 02 / 19$ & $04 / 09 / 19$ & Referral \\
\hline \multicolumn{8}{|l|}{ BUN } \\
\hline (mg/dl) & 28 & 25 & 19 & 14 & & 12 & $7-18$ \\
\hline SCr & & & & & & & $0.6-1.3$ \\
\hline$(\mathrm{mg} / \mathrm{dL})$ & 2.01 & 2,32 & 1.71 & 0.96 & & 0.75 & \\
\hline Albumin & & & & & & & $3.4-5.0$ \\
\hline$(g / d L)$ & 3.3 & & & 3,0 & & & \\
\hline \multirow[t]{2}{*}{$\operatorname{AST}(\mathbf{I U} / \mathbf{L})$} & & & & & & & $0-50$ \\
\hline & 294 & 612 & 299 & 149 & 262 & 79 & \\
\hline \multirow[t]{2}{*}{ ALT (IU/L) } & & & & & & & $0-50$ \\
\hline & 183 & 181 & 461 & 348 & 158 & 104 & \\
\hline $\mathrm{Na}$ & & & & & & & $136-145$ \\
\hline$(\mathbf{m m o l} / \mathbf{L})$ & 135 & & 139 & 136 & 136 & 139 & \\
\hline \multirow[t]{2}{*}{$\mathbf{K}(\mathbf{m m o l} / \mathbf{L})$} & & & & & & & $3.5-5.1$ \\
\hline & 3.6 & & 3.8 & 4.0 & 4.2 & 3.8 & \\
\hline \multirow[t]{2}{*}{$\mathrm{Cl}(\mathrm{mmol} / \mathrm{L})$} & & & & & & & 98-107 \\
\hline & 102 & & 103 & 100 & 98 & 101 & \\
\hline RBG & & & & & & & $N:<100$ \\
\hline$(\mathbf{m g} / \mathbf{d L})$ & 131 & & & & 79 & 101 & DM:>=126 \\
\hline Phosphate & & & & & & & $2.5-4.9$ \\
\hline$(\mathbf{m g} / \mathbf{d L})$ & & & 3.8 & & & & \\
\hline $\mathbf{L D H}(\mathbf{U} / \mathbf{L})$ & & & 447 & & & & $100-190$ \\
\hline \multicolumn{8}{|l|}{ Calcium } \\
\hline$(\mathbf{m g} / \mathbf{d L})$ & & & 7.4 & & & & $8.5-10.1$ \\
\hline \multicolumn{8}{|l|}{ Alkaline } \\
\hline Phosphatase & & & 53 & & & & $46-116$ \\
\hline \multicolumn{8}{|l|}{$(\mathbf{U} / \mathbf{L})$} \\
\hline CPK/CK & & 1183.1 & & & & & $<171 \mathrm{U} / \mathrm{L}$ \\
\hline
\end{tabular}




\section{Management:}

The patient is treated with Bromocriptine $2.5 \mathrm{mg}$ every 24 hours. Administration of Aminofluid infusion of $500 \mathrm{ml}$ in 24 hours, infusion of $0.9 \% \mathrm{NaCl}$ $1500 \mathrm{ml}$ in 24 hours. Administration of Ranitidine injection $50 \mathrm{mg}$ every 12 hours, Paracetamol tablets $500 \mathrm{mg}$ every 8 hours, Melopram $2 \mathrm{mg}$ every 24 hours (night). Antipsychotic therapy was postponed.

\section{Discussion}

Neuroleptic malignant syndromesyndrome is athat occurs as a result of serious complications of the use of antipsychotic drugs, but is rarely found. Characteristics that can be found in NMS are hyperthermia, rigidity, autonomic dysregulation and altered consciousness. Based on pathophysiology, NMS as a secondary effect of decreased activity of dopamine (DA) in the central nervous system, resulting in inhibition of dopamine type 2 (D2 receptors) and decreased amount of DA, in 3 central dopaminergic pathways; nigrostriatal, mesolimbic/cortical, hypothalamic. Constraints onpathway nigrostiatal and hypothalamic is believed to provide the onset of symptoms of NMS(1). Types of drugs associated with NMS can be seen in Table 3(1).

Table 3. Drugs Associated with NMS

$\begin{array}{ll}\text { Levodopa (L-DOPA) } & \begin{array}{l}\text { Neuroleptics: Phenothiazine, butyrophenones, } \\ \text { thiothixanes }\end{array}\end{array}$

Tolcapone/entacopone

Dopamine agonists

Amantadine
Atypical antipsychotics : olanzapine, risperidone, quetiapine metoclopramide, droperidol, prochlorperazine.

Amphetamines, cocaine, loxapine, diatrizoate, lithium, citalopram. 
This patient was found to have a history of risperidone and clozapine therapy after 12 years of treatment with haloperidol. Risperidone and clozapine are dopamine agonists that reduce the stimulation of postsynaptic D2 receptors in the nigrostriatal area, causing NMS symptoms.

The onset of NMS is quite rapid. Some patients develop NMS after receiving a single large dose of a neuroleptic, some patients develop NMS after 1-3 days of starting neuroleptic use, and most patients develop symptoms within 4-14 days of starting neuroleptic use(2).

Establishing a diagnosis in NMS cases requires a careful history, especially regarding the history of use of neuroleptic drugs, route of administration, and duration of administration. In addition to history taking, physical examination and supporting examinations are also needed to establish the diagnosis. NMS is a syndrome that rarely occurs but has the potential to be life-threatening, so prompt diagnosis followed by appropriate treatment will determine the patient's prognosis. The four classic symptoms of NMS include motor symptoms (muscle rigidity, bradykinesia, dystonia, mutism, chorea, tremor), hyperthermia, altered mental status (confusion, delirium, stupor, coma), and autonomic instability (respiratory irregularity, cardiac arrhythmias, elevated blood pressure). unstable, diaphoresis). Typical laboratory tests are an increase in the enzyme creatinine kinase (CK) above $1000 \mathrm{IU} / \mathrm{L}$ (more than $90 \%$ of cases), and can reach up to $100000 \mathrm{IU} / \mathrm{L}$, leukocytosis in the range $10000-40000 / \mathrm{mm} 3$, a mild increase in the enzyme lactate dehydrogenase ( LDH), liver function abnormalities (elevated SGOT/SGPT). Myoglobinuria can be caused by the process of rhabdomyolysis which can cause acute kidney failure $(3,4)$.

In this case, the diagnosis of NMS uses Levenson's criteria, which requires 3 major symptoms or 2 major symptoms with 4 minor symptoms. 1,10 In accordance with Levenson's criteria, this case shows symptoms of fever, rigidity, elevated CPK enzymes, changes in consciousness, diaphoresis, and leukocytosis. In addition, there are other criteria that can be used to diagnose SNM, namely the diagnostic criteria based on DSM-IV, namely: A. The occurrence of muscle rigidity and an increase in 
body temperature associated with the use of neuroleptic drugs B. Two or more of the following symptoms: Diaphoresis, increased blood pressure or unstable, tachycardia, incontinence, dysphagia, mutism, tremor, altered mental status, from confusion to coma, leukocytosis, and laboratory evidence of muscle lesion (elevated CK enzyme) C. Symptoms in criteria A and B are not attributable to other substances, another medical, or neurologic condition D. The symptoms in criteria A and B are not attributable to a mental disorder. Establishing the diagnosis of SNM using DSM-IV criteria requires 2 of criteria $A$ and at least 2 of criteria $B$. This case showed symptoms of fever, rigidity, elevated CPK enzymes, tachycardia, changes in mental status, diaphoresis and leukocytosis(1,5).

Management of SNM is based on clinical presentation, but the first step is discontinuation of neuroleptic drugs that are thought to trigger the onset of this syndrome. Empirical therapy such as bromocriptine and dantrolene. Bromocriptine is a dopamine agonist, while dantrolene is a muscle relaxant that works by inhibiting the release of calcium from the sarcoplasmic reticulum. Bromocriptine is useful for reversing hypodopaminergic conditions and is given orally or by a nasogastric tube (NG), starting with a dose of 2.5 mg 2-3 times per day and increasing the dose by 2.5 mg every 24 hours until a response occurs or until the maximum dose is reached, 45 $\mathrm{mg}$ per day. Bromocriptine is generally maintained for at least 10 days in cases of NMS with oral neuroleptics and at least 2-3 weeks for injectable neuroleptics. Therapy should not be discontinued suddenly even if clinical symptoms improve because it can lead to relapse of NMS. In this case, supportive therapy was carried out with immediate discontinuation of neuroleptic drugs, paracetamol tablets $500 \mathrm{mg}$ every 8 hours, intravenous fluid administration of $0.9 \% \mathrm{NaCl} 1500 \mathrm{ml}$ per 24 hours. Specific pharmacological therapy with bromocriptine at a dose of $2.5 \mathrm{mg}$ per 24 hours. The family was educated that bromocriptine was continued until 14-21 days after the symptoms of NMS disappeared and then gradually reduced. The choice of bromocriptine preparations refers to the patient's medical condition and the availability of bromocriptine which is easier to obtain. 
The mortality rate for NMS cases is $>30 \%$, but if treated as soon as possible the mortality rate will decrease to $10 \%$. If recognized early and treated immediately, usually NMS is not fatal and most patients will recover completely within 2-14 days. However, if diagnosis and treatment is delayed, healing may take several weeks or longer, and survivors may develop residual catatonia or parkinsonism or morbidity associated with renal or cardiopulmonary complications.

\section{Conclusion}

Neuroleptic malignant syndrome (NMS) is a potentially life-threatening neurological emergency syndrome associated with the use of neuroleptic drugs. This syndrome can be fatal and the mortality rate ranges from $5-20 \%$ if not treated properly. Death is usually caused by complications of arrhythmia, DIC, heart failure, respiratory failure, and kidney failure. In schizophrenic patients with initiation, dose increases need to be monitored with CPK examination to prevent the risk of developing NMS. Management of NMS is related to discontinuation of neuroleptic drugs that are suspected of triggering this syndrome, supportive therapy, correction of metabolic factors if abnormalities are found.

\section{Conflict of Interest}

The authors declare no conflict of interest. 


\section{REFERENCES}

1. Bhandari G. Neuroleptic malignant syndrome. Chapter 118. Available from: Www.apiindia.org/medicine_update_2013/chap118.pdf

2. Berman B D. Neuroleptic malignant syndrome: A review for neurohospitalists. Neurohospitalist. 2011;1(1):41-7.3

3. Nisijima K, Shioda K. Temporal changes in serum creatine kinase concentration and degree of muscle rigidity in 24 patients with neuroleptic malignant syndrome. Neuropsychiatrist Dis Treat. 2013;9:853-9.

4. Tsai MC, Huang TL. Severe neuroleptic malignant syndrome: Successful treatment with high-dose lorazepam and diazepam: A case report. Chang Guang Med J. 2010;33(5):9.

5. Levenson JL. Neuroleptic malignant syndrome. Am J Psychiatry. 1985;142(10):1137-45. 